Research Article

\title{
Stent Applications for Palliative Treatment in Advanced Stage Esophageal Cancers
}

\author{
Mustafa Şentürk (D), Murat Çakır ${ }^{(D}$, Mehmet Aykut Yıldırım (D), and Ömer Kişi \\ Department of General Surgery, Necmettin Erbakan University Meram Medicine Faculty, Turkey \\ Correspondence should be addressed to Mustafa Şentürk; m-sntrk@hotmail.com
}

Received 1 September 2021; Revised 8 September 2021; Accepted 25 September 2021; Published 18 October 2021

Academic Editor: James H. Tabibian

Copyright (c) 2021 Mustafa Şentürk et al. This is an open access article distributed under the Creative Commons Attribution License, which permits unrestricted use, distribution, and reproduction in any medium, provided the original work is properly cited.

\begin{abstract}
Background and Aim. Endoscopic stenting is a generally safe and effective palliative treatment for esophageal malignancies. In this study, we aimed to present endoscopic stent applications, adverse events, and relative advantages of covered versus uncovered stents in our center. Methods. We examined cases of endoscopic stenting for palliative treatment of advanced stage esophageal cancers between January 2014 and July 2019. Age, gender, location of mass, adverse events, survival time, and stent type were evaluated. Outcomes of fully covered and uncovered self-expanding stents were compared with regard to adverse events, including stent migration and occlusion. Results. The mean age of the patients was $66.4 \pm 1,52$ were male, and 8 were female. Patients were followed up for a mean of 133 days. The most common complication due to stenting was migration. 13 patients developed adverse events. Migration was the most common adverse event, occurring in 8 (13\%) patients. Although the migration rate of fully covered stents was higher than uncovered stents, there was no statistically significant difference $(p=0.47)$. Stent occlusion was observed in 4 patients. In three cases, it was due to the tumor; an uncovered stent was placed again in these cases. Food-related occlusion developed in one patient. There was no statistical difference in terms of overall adverse event rate when comparing fully covered stents to uncovered stents $(p=0.68)$. Conclusion. Endoscopic stenting is a viable palliative method with low morbidity and mortality in experienced centers. Though there are relative advantages with covered versus uncovered stents in individual cases, the overall adverse event rate is low and relatively similar.
\end{abstract}

\section{Introduction}

The number and breadth of endoscopic procedures performed continues to increase. Endoscopy is used universally in the diagnosis and treatment of many diseases. Endoscopic stenting has been increasingly used in the palliation of gastrointestinal malignancies [1]. Stenting provides a relatively easy and effective palliative treatment in patients with metastatic or advanced esophageal cancer.

Endoscopic stenting has been increasingly used in the palliation of gastrointestinal malignancies [1]. Stent insertion provides an easy and effective treatment exclusively in patients with metastatic or advanced esophageal cancer. Endoscopic stenting is a different method for the treatment of anastomosis leakage and esophageal fistula. Stent procedure in distal esophageal malignant stenosis is a simple and uncomplicated treatment method for the patient to relieve correct oral intake and dysphagia [2]. A similar accomplishment is partly achieved in proximal esophageal strictures [3]. Esophageal stent practiced in malignant stenosis can still be successfully practiced in benign stenosis [4]. There are numerous types of self-expandable stents (such as self-expandable biodegradable stents and self-expandable plastic stents). The use of SEMS has been on the increase. Uncovered (UC), semicovered, and fully covered (FC) stents are produced for use in different indications [5]. FC stents are used for anastomotic leakage and fistula. FC stents used for benign diseases can be removed if desired. UC stents are mostly preferred in malignant stenosis. Various complications related to the procedure concur with the use of endoscopic stents. Migration, fistula formation, bleeding, and occlusion are among the most common ones [4]. 
This study is aimed at discussing the results of selfexpandable metal stent (SEMS) applications that we use for palliative treatment in patients with advanced esophageal cancer, in the light of the literature.

\section{Methods}

Study was made in the 1400-bed Necmettin Erbakan University Meram Medical Faculty Hospital in the Central Anatolian region of Turkey. Patients who underwent stenting for palliative treatment in our center due to advanced stage esophageal cancer between January 2014 and June 2019 were evaluated with case series analysis. The research was conducted according to the WMA Declaration of HelsinkiEthical Principles for Medical Research Involving Human Subjects. The study was approved by the local ethics committee. 60 patients were included in our study. Patients with stent implantation due to benign esophageal stricture and postoperative leakage were excluded from the study. The type of stent was determined according to the indication and localization and size of the lesion. The stent length was determined upon endoscopy. The stent was used in stenoses that did not allow the passage of the scope. In occlusive lesions, the length of the stent was determined by imaging methods adjusting the length of the stent accordingly. We preferred uncovered (UC) stents for tumoral occlusion. Fully covered (FC) stents were preferred for the cases of fistula formation. In our clinic, stents with a length of $10-12 \mathrm{~cm}$ and a width of 20 french are used.

All endoscopic procedures were performed by 3 general surgeons in the general surgery clinic. All procedures were performed under anesthesia. The stents were inserted with guidewire under endoscopic control (Figure 1). In cases with in occlusive lesions where the endoscope was inapplicable, dilatation was performed first. 24 hours after the procedure, control radiographs were taken using X-ray. Oral intake was initiated following the X-ray control. Age, gender, location of mass, complications, survival time, and stent type of the patients were evaluated. Both stents (FC and UC) were compared for overall complication, occlusion, and migration development.

2.1. Statistical Analysis. The computer software used for biostatistical analysis was Statistical Package for the Social Sciences (SPSS 21 Inc., Chicago, IL, USA). Categorical variables were presented as frequency (percentage), and continuous variables were reported as mean \pm standard deviation. Differences in patients' characteristics between FC and UC stents were examined by Pearson's chi-square test for categorical variables.

\section{Results}

Demographic data are given in Table 1 . Sixty patients underwent stent insertion. The number of stents was 70 . The mean age was $66.4 \pm 16$. Ten (16\%) patients underwent multiple endoscopic stent placement. The stent was successfully inserted in all patients (Figure 1). Oral intake improved in all patients (completely in $75 \%$ and partly in $25 \%$, respec-

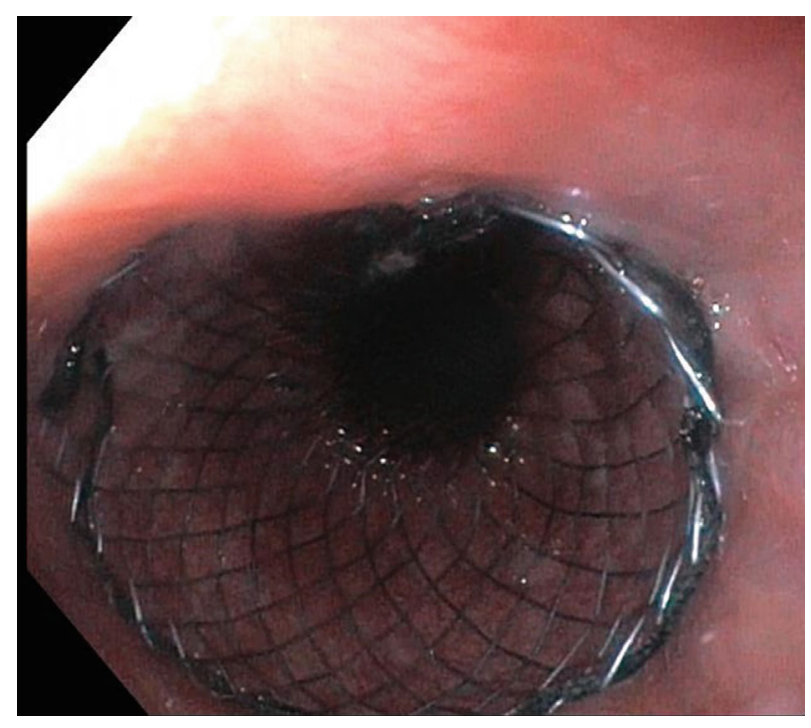

Figure 1: Stent placed in the esophagus.

tively). The mean follow-up period was $133 \pm 130$ days. The mean length of hospital stay was 2.1 days [1-5].

13 patients developed complications. Migration was the most common complication after stenting. It occurred in 8 (13\%) patients who underwent stent placement. In $3(5 \%)$ of these patients, the stent was placed back to its previous position. It was applied especially in patients with migration occurring within a few days. In four (6\%) patients, the stents were removed and changed with new ones. Only 1 patient developed a fatal complication. The patient died in the second postoperative month due to mediastinitis due to perforation. Stent migration occurred in 2 patients after chemotherapy (Figure 2). These patients were those who underwent FC stenting due to tracheoesophageal fistula. The old stent was removed, and a new one was placed.

Three patients (5\%) developed hypotension during the procedure, and the procedure had to be interrupted. These patients had poor general status and apparent malnutrition. The procedure was successfully performed the next day.

One of the complications related to the stent is occlusion. It was seen in 4 patients. In three cases, occlusion due to a tumor was seen after 3 months. UC stent was placed again in these cases due to tumor growth. Food-related occlusion was observed in one patient and was removed endoscopically. Although the migration rate of FC stents was higher than UC stents, there was no statistically significant difference $(p=0.47)$. There was no statistical difference in terms of complications when FC stents and UC stents were compared $(p=0.68)$ (Table 2 ).

\section{Discussion}

In both malignant and benign UGI (upper gastrointestinal) tract occlusion, treatment with SEMS is considered to be a safer, less invasive, and effective method than oncological treatments and surgical. SEMS also reduces the rate of complications and length of hospital stay. In recent years, its use has increased as SEMS has a lower morbidity and 
TABLE 1: Demographic characteristics of patients $(n=60)$.

\begin{tabular}{lccr}
\hline & $n$ (patient) & $\%$ & Mean \pm SD \\
\hline Sex & & & \\
Male & 52 & 86 & \\
Female & 8 & 14 & \\
Age (year) & & & $66.4 \pm 16$ \\
Survival (month) & & & $4.4 \pm 4.3$ \\
Stent & & & \\
Fully covered & 10 & 16.7 & \\
Uncovered & 50 & 83.3 & \\
Location of mass & & & \\
Proximal esophageal ca & 18 & 30 & \\
Cardioesophageal junction tumor & 42 & 70 & \\
Complication & & & \\
Migration & 8 & 13 & \\
Occlusion & 4 & 6 & \\
Perforation & 1 & 2 & \\
\hline
\end{tabular}

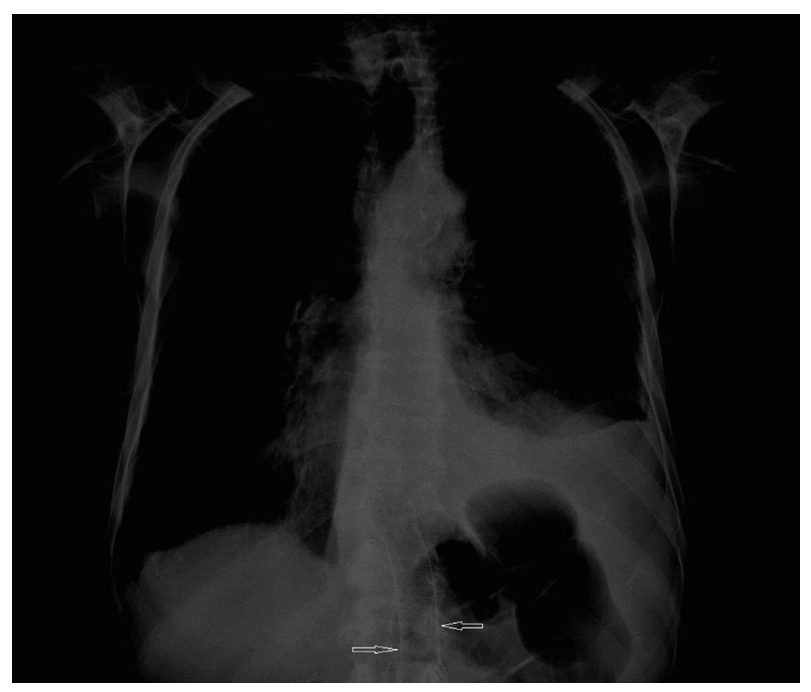

FIGURE 2: Covered stent migration.

TABle 2: Compare of stent types.

\begin{tabular}{lccc}
\hline & $\begin{array}{c}\text { Fully covered stent } \\
(n=10)\end{array}$ & $\begin{array}{c}\text { Uncovered stent } \\
(n=50)\end{array}$ & $\begin{array}{c}p \\
\text { value }\end{array}$ \\
\hline Complication & 4 & 9 & 0.68 \\
Migration & 3 & 5 & 0.47 \\
Occlusion & 1 & 3 & 0.52 \\
\hline
\end{tabular}

mortality rate compared to conventional methods [5]. In this study, we shared our SEMS experiences in single center esophageal malignant occlusions. $30 \%$ of the cases were located in the proximal esophagus and $70 \%$ in the cardioesophageal junction.

While FC SEMS sees more migration, tumor growth is more common in cases with UC stent $[6,7]$. We prefer FC stents more frequently due to their complete isolation, par- ticularly in the fistulae, and easy removal. Migration occurred in 4 of 10 cases in which we applied a FC stent. The stents were placed back to their previous position. Stents that fell into the gastric cavity were removed and replaced with new ones. Rarely, stents were fixed with a hemostatic clip.

Most tracheoesophageal fistulas arise from locally advanced malignancy. In such cases, a covered metallic stent is applied for palliative treatment $[8,9]$. Fully covered SEMS placement during the early term and minimally invasive drainage is an effective and safe treatment option [10]. In our series, Only 1 patient presented with fatal complications. In the second postoperative month, the patient died because of mediastinitis due to perforation. The occlusion was observed in 4 (6\%) cases. They are advantageous as it is easier to remove them once the disease is treated. We mostly preferred FC stents in our cases with fistula formation. The handicap of using this type was a higher rate of migration. Although the migration rate of FC stents was higher than UC stents, there was no statistically significant difference $(p=0.47)$. Consequently, it resulted in a higher number of endoscopic interventions.

Oral intake is corrected in more than $95 \%$ of patients undergoing stent insertion due to occlusion $[11,12]$. The accomplishment rate in fistula cases changes between $70 \%$ and $100 \%$ [13]. Stent migration, overgrowth, or ingrowth should be considered in patients presenting with dysphagia after oral intake was previously corrected. Dysphagia was corrected in all of our cases. Occlusion was observed due to tumor ingrowth in three patients. A second stent was inserted to solve these problems. One patient had a foodrelated occlusion, which was corrected by the endoscopic intervention. Other studies have demonstrated technical success rates (defined as successful insertion and adequate placement of the stent) of 83 to $100 \%$ and clinical success rates (defined as palliation of dysphagia) of 80 to $95 \% 14$. In our series, technical success was achieved in SEMS procedures $(100 \%)$. Dysphagia improved in all our patients. However, $25 \%$ of the cases could not tolerate solid food and only tolerated liquid food. Before the stent was placed, all patients had liquid or solid food intolerance. Oral intake was provided after stent placement in all patients.

Although tumor internal growth rates of FC stents are reported to be lower than those of UC stents, migration rates are higher, particularly in the gastroesophageal junction, due to their limited adhesion ability. However, it is reported that short and thinner caliber stents can migrate more. In our series, the stent calibers were the same $(20 \mathrm{~mm})$. Stent migration is reported to occur in 10 to $25 \%$ of the coated stents and 2 to $5 \%$ of the UC stents [14]. The migration rate in our study was 30\% in FC stents and 10\% in UC stents, and our migration rate was $13 \%$ in all cases. Migration rate was higher compared to the literatüre. We think that this situation is caused by the termination of the procedure without waiting for the full opening of the stent during the procedure or the wrong stent selection. Neoadjuvant or palliative chemoradiotherapy is thought to increase the rate of stent migration [15]. Two of our patients had migration after chemoradiotherapy. When FC stents and UC stents were 
compared, there was no statistically significant difference in terms of complications $(p=0.49)$.

Reocclusion usually occurs as a result of tumor overgrowth or food impaction, and its incidence is reported to be between 3 and 15\% for covered and 10 and $42 \%$ for uncovered stents [16]. Stents covered with 5-fluorouracil or paclitaxel (drug-eluting stents) have been introduced to prevent tumor ingrowth in recent studies [17]. In this study, food-related occlusion was observed in 1 case and tumor ingrowth occlusion in 3 cases $(6 \%)$.

Migration, occlusion, perforation, hemorrhage, and ulceration are the most widespread complications related to stents. Mortality rate stent application varies between $0.5 \%$ and $2 \%$ [ 18,19$]$. Complications can be categorized under intraoperative or postoperative complications in the early and late periods. Timing of chemotherapy, stent length, and tumor stage is important parameters in the development of complications $[20,21]$. Thirteen of our cases developed complications. Most of them were corrected with small interventions. Mortality was determined as $1 \%$. However, our complication rate is higher compared to the literature. We attributed this situation to the long and strict followup period.

\section{Conclusions}

We found that there was no difference between stent types in terms of complication development among patients undergoing palliative endoscopic stenting of advanced esophageal cancers. Endoscopic stenting in this setting has low mortality and morbidity and is effectively in reducing dysphagia. The endoscopist must be experienced and prepared to address complications should they arise.

\section{Data Availability}

The data used to support the findings of this study are available from the corresponding author upon request.

\section{Ethical Approval}

Ethics committee approval was received for this study from the ethics committee of Necmettin Erbakan University Meram School of Medicine (IRB Number: 2021/3031).

\section{Conflicts of Interest}

Drs Mustafa Şentürk, Murat Çakır, Mehmet Aykut Yıldırım, and Ömer Kişi have no conflicts of interest or financial ties to disclose.

\section{Authors' Contributions}

Study planning was contributed by MŞ and MÇ. Data collection and analysis were contributed by MAY, MŞ and ÖK. Data interpretation was contributed by MŞ, MÇ, MAY, and ÖK. Drafting of the manuscript was contributed by $\mathrm{MSS}$ and MÇ. Critical review of the manuscript was contributed by MŞ, MÇ, MAY, and ÖK. All authors approved the final version of the manuscript.

\section{References}

[1] R. Kochar and N. Shah, "Enteral stents: from esophagus to colon," Gastrointestinal Endoscopy, vol. 78, no. 6, pp. 913918, 2013.

[2] G. Battaglia, A. Antonello, S. Realdon, F. Cavallin, F. Giacomini, and S. Ishaq, "Feasibility, efficacy and safety of stent insertion as a palliative treatment for malignant strictures in the cervical segment of the esophagus and the hypopharynx," Surgical Endoscopy, vol. 30, no. 1, pp. 159-167, 2016.

[3] E. Speer, C. M. Dunst, A. Shada, K. M. Reavis, and L. L. Swanström, "Covered stents in cervical anastomoses following esophagectomy," Surgical Endoscopy, vol. 30, no. 8, pp. 3297-3303, 2016.

[4] P. Sharma and R. Kozarek, "Role of Esophageal Stents in Benign and Malignant Diseases," American Journal of Gastroenterology, vol. 105, no. 2, pp. 258-273, 2010.

[5] H. Bektaş, B. Gürbulak, Y. Düzköylü et al., "Clinical outcomes of upper gastrointestinal stents and review of current literature," JSLS : Journal of the Society of Laparoendoscopic Surgeons, vol. 21, no. 4, 2017.

[6] T. DaVee, S. Irani, C. L. Leggett et al., "Stent-in-stent technique for removal of embedded partially covered self-expanding metal stents," Surgical Endoscopy, vol. 30, no. 6, pp. 23322341, 2016.

[7] N. Bethge, A. Sommer, and N. Vakil, "A prospective trial of self-expanding metal stents in the palliation of malignant esophageal strictures near the upper esophageal sphincter," Gastrointestinal Endoscopy, vol. 45, no. 3, pp. 300-303, 1997.

[8] J. H. Shin, H.-Y. Song, G.-Y. Ko, J.-O. Lim, H.-K. Yoon, and K.-B. Sung, "Esophagorespiratory fistula: long-term results of palliative treatment with covered expandable metallic stents in 61 patients," Radiology, vol. 232, no. 1, pp. 252-259, 2004.

[9] M. Spaander, T. Baron, P. Siersema et al., "Esophageal stenting for benign and malignant disease: European Society of Gastrointestinal Endoscopy (ESGE) Clinical Guideline," Endoscopy, vol. 48, no. 10, pp. 939-948, 2016.

[10] P. G. A. van Boeckel, A. Sijbring, F. P. Vleggaar, and P. D. Siersema, "Systematic review: temporary stent placement for benign rupture or anastomotic leak of the oesophagus," Alimentary Pharmacology \& Therapeutics, vol. 33, no. 12, pp. 1292-1301, 2011.

[11] I. Rozanes, A. Poyanli, and B. Acunaş, "Palliative treatment of inoperable malignant esophageal strictures with metal stents: one center's experience with four different stents," European Journal of Radiology, vol. 43, no. 3, pp. 196-203, 2002.

[12] P. Philips, D. A. North, C. Scoggins, M. Schlegel, and R. C. G. Martin, "Gastric-esophageal stenting for malignant dysphagia: results of prospective clinical trial evaluation of long-term gastroesophageal reflux and quality of life-related symptoms," Journal of the American College of Surgeons, vol. 221, no. 1, pp. 165-173, 2015.

[13] I. Raijman, I. Siddique, J. Ajani, and P. Lynch, "Palliation of malignant dysphagia and fistulae with coated expandable metal stents: experience with 101 patients," Gastrointestinal Endoscopy, vol. 48, no. 2, pp. 172-179, 1998.

[14] S. Y. Park, C. H. Park, S. B. Cho et al., "The usefulness of clip application in preventing migration of self-expandable metal stent in patients with malignant gastrointestinal obstruction," The Korean Journal of Gastroenterology, vol. 49, pp. 4-9, 2007.

[15] J. P. Im, J. M. Kang, S. G. Kim, J. S. Kim, H. C. Jung, and I. S. Song, "Clinical outcomes and patency of self-expanding metal 
stents in patients with malignant upper gastrointestinal obstruction," Digestive Diseases and Sciences, vol. 53, no. 4, pp. 938-945, 2008.

[16] Q. Guo, S. Guo, and Z. Wang, "A type of esophageal stent coating composed of one 5-fluorouracil-containing EVA layer and one drug-free protective layer: in vitro release, permeation and mechanical properties," Journal of Controlled Release, vol. 118, no. 3, pp. 318-324, 2007.

[17] H. W. Kang and S. G. Kim, "Upper gastrointestinal stent insertion in malignant and benign disorders," Clinical Endoscopy, vol. 48, no. 3, pp. 187-193, 2015.

[18] V. S. Medeiros, B. C. Martins, L. Lenz et al., "Adverse events of self-expandable esophageal metallic stents in patients with long-term survival from advanced malignant disease," Gastrointestinal Endoscopy, vol. 86, no. 2, pp. 299-306, 2017.

[19] T. H. Baron, "Minimizing endoscopic complications: endoluminal stents," Gastrointestinal Endoscopy Clinics of North America, vol. 17, no. 1, pp. 83-104, 2007.

[20] L. Fuccio, M. Scagliarini, L. Frazzoni, and G. Battaglia, "Development of a prediction model of adverse events after stent placement for esophageal cancer," Gastrointestinal Endoscopy, vol. 83, no. 4, pp. 746-752, 2016.

[21] A. Laquière, P. Grandval, D. Heresbach et al., "Self-expanding plastic stent removed after radiochemotherapy for advanced esophageal cancer," Diseases of the Esophagus, vol. 27, no. 2, pp. 176-181, 2014. 